\title{
SUBARACHNOID HAEMORRHAGE RESULTS IN POOR QUALITY OF LIFE INDEPENDENT OF PITUITARY HORMONE CONCENTRATIONS
}

Zainab G. Nagras, M. Klose and U. Feldt-Rasmussen

Department of Medical Endocrinology, University Hospital of Copenhagen, Rigshospitalet, Copenhagen, Denmark
Corresponding author: z.nagras@gmail.com

\section{INTRODUCTION}

Subarachnoid haemorrhage $(\mathrm{SAH})$ is associated with a mortality rate of $30 \%$ and the survivors face significant morbidity including fatigue, isolation, depression and sleep disorders; factors affecting general health related quality of life (QoL). QoL is also known to be decreased in pituitary-hypothalamic insufficiency and tends to improve after treatment. SAH has been suggested to be a common cause of hypopituitarism by some whereas not by others. We aimed to investigate if aneurismal SAH results in changes in QoL and if these changes relate to the concentrations of pituitary hormones.

\section{METHODS}

We performed a cross-sectional evaluation including a subgroup with prospective follow-up. QoL measurements were performed at 12 months post$\mathrm{SAH}$ in 62 patients with $\mathrm{SAH}$ and 30 healthy controls. Twenty-six of the patients were followed prospectively (3-, 6-, and 12 months) post-SAH. Full anterior pituitary assessments, including stimulation tests for growth hormone and adrenal insufficiencies, were performed on collected blood samples at all time points. QoL evaluation was assessed by using two generic questionnaires, the euroQoL-5D (EQ-5D) and Nottingham Health Profile (NHP) and one specific of growth hormone deficiency (GHD), the Quality of Life Assessment of Growth Hormone Deficiency in Adults (QoL-AGHDA).

\section{RESULTS}

Post-SAH patients scored significantly lower on all QoL questionnaires (NHP, EQ-5D and QoL-AGHDA) when compared to controls 12 months post-SAH (all patients, figure 1), as well as at 3-, 6-, and 12 months (prospective subgroup).
Figure 1: Mean QoL - patients vs. controls - 12 months post-SAH

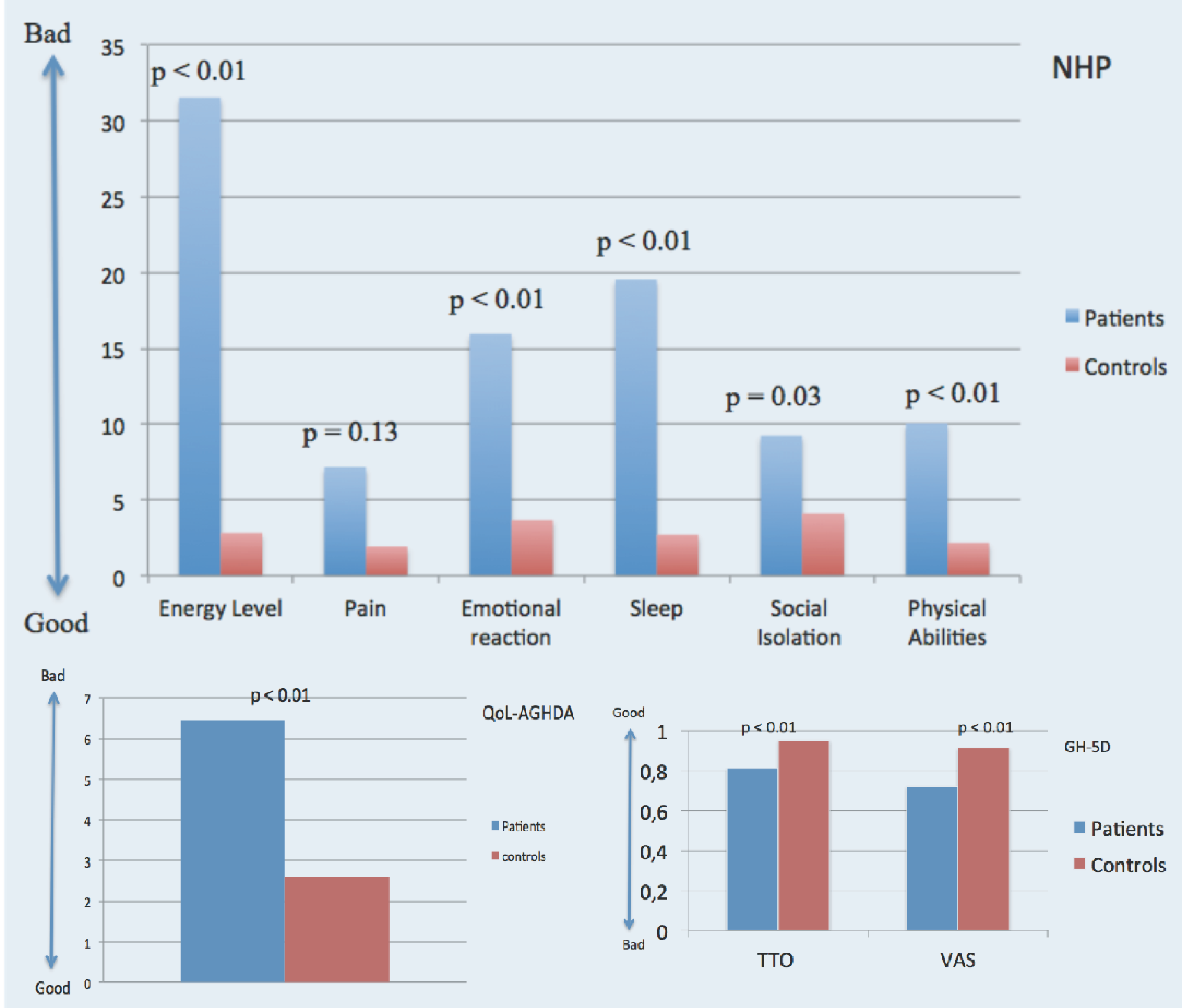

Pituitary hormone concentrations at 12 months were found to be within the normal range. No correlations between QoL measurements and pituitary hormone levels were found.

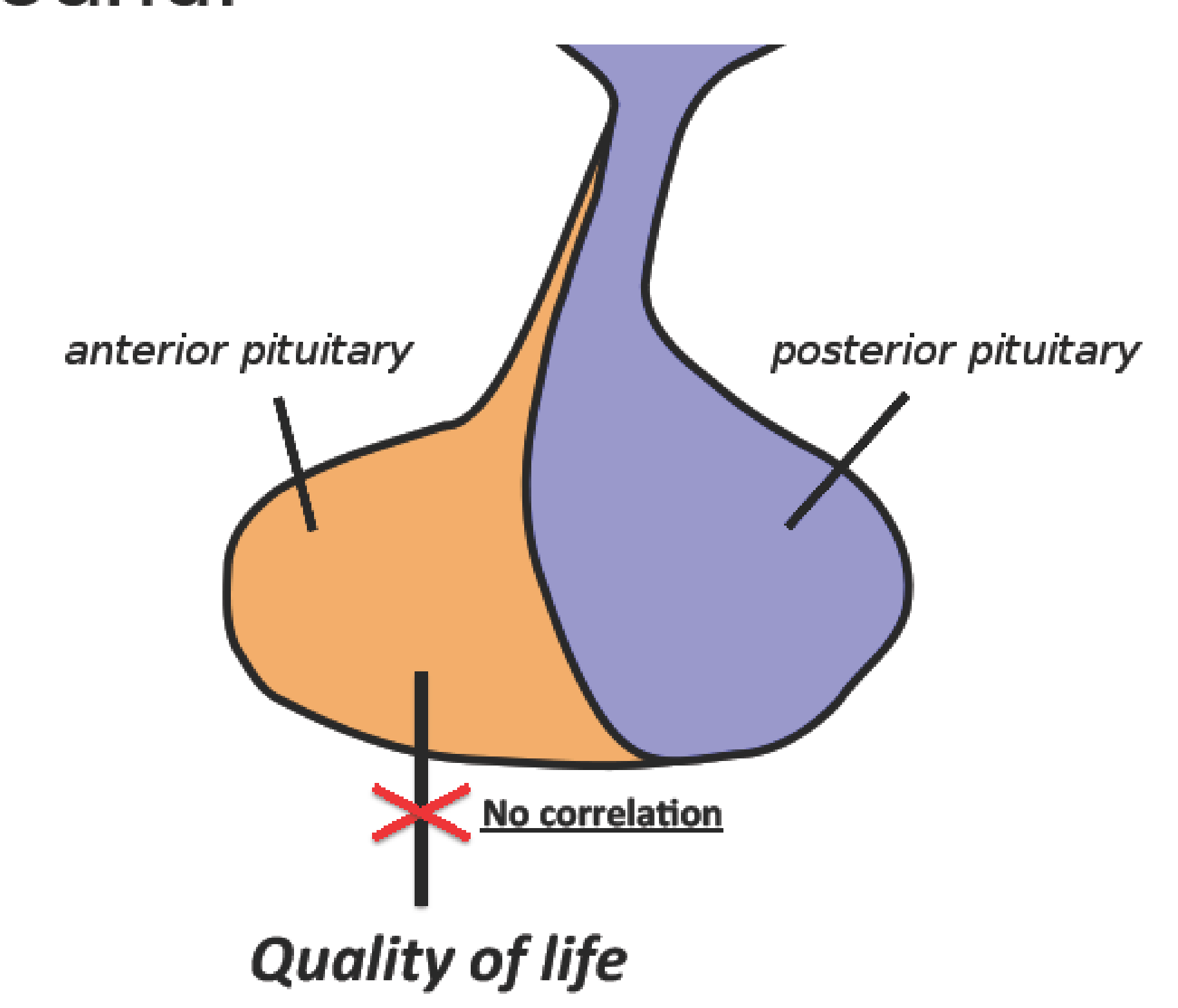

Likewise there were no significant differences in QoL between patients with the lowest and highest hormone levels, respectively ( $p>0.08$ for all comparisons).

\section{CONCLUSION}

$\mathrm{SAH}$ is associated with long term worsened QoL, but it appears to be independent of pituitary hormone concentration, and thus rather a consequence of the SAH per se. 